\title{
Análisis Semántico y Gramatical de los Términos Latinos de los Ramos Terminales del Nervio Nasociliar
}

\author{
Semantic and Grammatical Analysis of Latin Terms \\ of the Terminal Branches of the Nasociliary Nerve
}

Marco Guerrero ${ }^{1,3}$; Juan Ocampo ${ }^{2,3}$; María P. Moya ${ }^{3,5}$ \& Clivia Guerrero ${ }^{3,4,6}$

GUERRERO, M.; OCAMPO, J.; MOYA, M. P. \& GUERRERO, C. Análisis semántico y gramatical de los términos latinos de los ramos terminales del nervio nasociliar. Int. J. Morphol., 37(1):71-75, 2019.

RESUMEN: La necesidad de unificar criterios respecto a los nombres de las estructuras anatómicas ha sido una permanente preocupación de los anatomistas del mundo, de tal manera que a partir de 1895 se inicia un proceso de estandarización y normalización de la terminología anatómica mundial. Se publica la Nomina Anatomica tratando de nominar las estructuras con un solo nombre en latín y se suprime los epónimos y homónimos. En la actualidad la Terminologia Anatomica sustituye a la Nomina Anatomica, con las mismas características, pero con la adición del término en el idioma de cada país. Sin embargo, persisten algunos errores desde la elaboración de la Nomina Anatomica y que se mantienen en Terminologia Anatomica, derivados tanto de la estructura gramatical latina, principalmente en el número y género, así como de la descripción de algunas estructuras anatómicas. Este es el caso de los ramos del nervio nasociliar, específicamente del ramo etmoidal anterior y del ramo infratroclear. Para el efecto se realizó una revisión de la descripción del nervio nasociliar y sus ramos terminales, se compararon entre sí y con los nombres que aparecen en la Terminologia Anatomica, para verificar que tanto la descripción como la construcción gramatical latina sean correctas. Se encontraron errores en la estructuración gramatical y jerárquica del ramo nasal interno, así como la supresión de los ramos palpebrales superior e inferior del nervio infratroclear, por lo que proponemos el cambio del término codificado con A14.2.01.031 a Ramus nasalis internus y la adición de los nombres Ramus palpebralis superior y Ramus palpebralis inferior.

PALABRAS CLAVE: Terminologia Anatomica; Nervio nasociliar; Nervio etmoidal anterior; Nervio infratroclear.

\section{INTRODUCCIÓN}

La necesidad de unificar los términos anatómicos utilizados por los anatomistas alrededor del mundo, de tal manera que su referencia sea única y comprendida por todos, dio lugar para que, convocados por la Sociedad Alemana de Anatomía, éstos se reunieran y decidieran elaborar un documento que compilara y unificara los nombres de las estructuras anatómicas descubiertas hasta la época. El trabajo fue encargado a Wilhelm His y sus colaboradores Kölliker, Hertwig, Kollmann, Merkel, Swallow, Toldt, Waldeyer, actuando como secretario, Bardelebe (His, 1895); y que culminó con la aprobación y posterior publicación de la Nomina Anatomica de Basilea.

Varias fueron las reuniones y congresos que se realizaron posteriormente para revisar Nomina Anatomica, así en 1903 en Nancy (Francia), se propuso la creación de la Federación Internacional de Asociaciones de Anatomía (FIAA). En 1935 se revisa y modifica Nomina Anatomica existente, a la que se denominó Jena Nomina Anatomica. (Acuña et al., 2007; Álvarez Guisbert \& Campohermoso Rodríguez, 2007).

En 1950, en Oxford se estableció el Comité de la Nomenclatura Anatómica Internacional (IANC), y en 1955 en París, se acordó adoptar una nomenclatura latina basada en la Basilea Nomina Anatomica (BNA). Fue denominado: Paris Nomina Anatomica (PNA).

El Comité Internacional de Nomenclatura Anatómica publicó en 1989 la sexta edición de la Nomina Anatomica

\footnotetext{
${ }^{1}$ Cátedra de Anatomía Normal, Facultad de Ciencias Médicas, Universidad Central del Ecuador, Quito, Ecuador.

${ }^{2}$ Cátedra de Histología Normal, Facultad de Ciencias Médicas, Universidad Central del Ecuador, Quito, Ecuador.

${ }^{3}$ Doctorado en Ciencias Morfológicas, Facultad de Medicina, Universidad de La Frontera, Temuco, Chile.

${ }^{4}$ Cátedra de Anatomía, Facultad de Ciencias de la Salud, Universidad de Las Américas, Quito, Ecuador.

${ }^{5}$ Facultad de Ciencias de la Salud, Universidad Autónoma de Chile, Temuco, Chile.

${ }^{6}$ Unidad de Medicina Legal Zona 9, Policía Nacional del Ecuador, Ecuador.
} 
sin someterla a la revisión de la FIAA ni a la aprobación del XIII Congreso celebrado en Río de Janeiro, esto causó un conflicto con Federación Internacional de Asociaciones de Anatomía, por lo que se crea el Comité Federativo de Terminologia Anatomica (FCAT). En 1998 se creó Terminologia Anatomica Internacional, que a diferencia de la Nomina Anatomica, no solamente incluye la terminología en latín, sino también en inglés, además proporciona una distribución semántica que permite una identificación más objetiva de la estructura anatómica, la que contempla la asignación de un código a cada término y representación de las relaciones entre las estructuras (Whitmore, 1999; Fabry et al., 2006; Vandaele \& Harvey, 2013).

En la configuración de la Nomina Anatomica y Terminologia Anatomica se establecen algunas similitudes, tales como el uso del latín como idioma universal para denominar las estructuras anatómicas, el valor informativo y descriptivo de las mismas y la supresión de epónimos y homónimos (Duque Parra et al., 2002; Álvarez Guisbert \& Campohermoso Rodríguez; Echeverría Pereda \& Jiménez Gutiérrez, 2010). Sin embargo, aún no se logra una armonización y estandarización completa de los términos en latín traducidos para los diferentes idiomas (Vásquez \& del Sol, 2015), e inclusive hay términos que tienen un uso incorrecto del caso, género y número, sobre todo en la segunda y tercera declinaciones (Marecková et al., 2001).

Bajo este hecho, se consideró necesario analizar los sustantivos y adjetivos utilizados en la Terminologia Anatomica (Comité Federativo sobre Terminología Anatómica; International Federation of Associations of Anatomists \& Sociedad Anatómica Española, 2001), para nominar a los ramos del nervio etmoidal anterior e infratroclear tomando como parámetros la descripción anatómica de los mismos, así como su estructura gramatical latina.

\section{MATERIAL Y MÉTODO}

Se realizó un análisis de Terminologia Anatomica publicada y aprobada por el Comité Federativo sobre la Terminología Anatómica (FCAT), correspondiente a los ramos del nervio nasociliar, derivado del nervio oftálmico, en cuanto a su estructura gramatical. Luego se buscó y comparó las descripciones de dichos nervios antes de la aprobación y publicación de la primera Nomina Anatomica en 1895 y luego de ella.

Para la selección de los textos de anatomía publicados antes de la aparición de la primera Nomina Anatomica, se determinó como criterio de inclusión el acceso virtual a los textos de anatomía gratuitos disponibles en la página web Open Library (2018), desde 1835, así como la relevancia de sus autores. Luego de la aparición de la primera Nomina Anatomica se seleccionó textos de anatomía de los autores clásicos en nuestro medio.

Los documentos se clasificaron en dos grupos: aquellos documentos escritos antes de la publicación de la primera Nomina Anatomica y los que aparecieron luego de la misma; en cada documento se analizó la descripción detallada sobre el nervio nasociliar, ramo del trigémino. En segundo lugar, se clasificaron los documentos que describían cada uno de los ramos terminales del nervio nasociliar, tanto en singular como en plural.

Finalmente se revisó la estructura gramatical de los nombres de los ramos terminales del nervio nasociliar en latín y se comparó con los nombres descritos en la primera Nomina Anatomica y Terminologia Anatomica.

\section{RESULTADOS}

En Terminologia Anatomica actual se observó que el nervio oftálmico (Nervus ophthalmicus), primera rama del trigémino que se encuentra con el código A14.2.01.016 tienes tres ramas principales que son el nervio nasociliar (Nervus nasociliaris), el nervio frontal (Nervus frontalis) y el nervio lagrimal (Nervus lacrimalis).

La codificación de los ramos del nervio nasociliar se puede observar en la Tabla I.

Cuando el nervio nasociliar llega próximo al foramen etmoidal anterior, se divide en sus ramos terminales: el nervio etmoidal anterior y el nervio infratroclear.

El nervio etmoidal anterior atraviesa el foramen etmoidal anterior y llega a la fosa craneal anterior, cruza la lámina cribosa del etmoides y desciende por la fisura etmoidal a la cavidad nasal, en donde se divide en dos ramos: ramo nasal medial, del cual se desprenden fibras nerviosas para el tabique nasal; y el ramo nasal lateral, del cual se desprenden fibras nerviosas para la parte anterior de la pared lateral de la cavidad nasal y tegumentos nasales.

El nervio infratroclear se dirige anteriormente entre los músculos oblicuo superior del ojo por arriba y el margen superior del músculo recto medial del ojo por abajo, e inferior a la polea de reflexión del músculo oblicuo superior del ojo se divide en dos ramos: palpebral superior y palpebral inferior. 
Tabla I. Codificación del nervio nasociliar. Tomado de Terminologia Anatomica (Comité Federativo sobre Terminología Anatómica; International Federation of Associations of Anatomists \& Sociedad Anatómica Española, 2001).

\begin{tabular}{llll}
\hline A14.2.01.025 & N. nasociliaris & Nasociliary nerve & N. nasociliar \\
\hline A14.2.01.026 & R. communicans cum ganglio ciliari; & Communicating Branch with ciliary & R. comunicante con el ganglio \\
& $\begin{array}{l}\text { Radix sensoria ganglii ciliaris; } \\
\text { Radix nasociliaris ganglii ciliaris. }\end{array}$ & ganglion; Sensory root of ciliary; & ciliar; Raíz sensitiva del ganglio \\
& & & Nasociliary root of ciliary ganglion. Raíz nasociliar del \\
ganglio ciliar.
\end{tabular}

$\begin{array}{ll}\text { A14.2.01.027 } & \text { Nn. ciliares longi } \\ \text { A14.2.01.028 } & \text { N. ethmoidalis posterior } \\ \text { A14.2.01.029 } & \text { R. meningeus anterior } \\ \text { A14.2.01.030 } & \text { N. ethmoidalis anterior } \\ \text { A14.2.01.031 } & \text { Rr. nasales interni } \\ \text { A14.2.01.032 } & \text { Rr. nasales laterales } \\ \text { A14.2.01.033 } & \text { Rr.nasales mediales } \\ \text { A14.2.01.034 } & \text { R. nasalis externus } \\ \text { A14.2.01.035 } & \text { N. infratrochlearis } \\ \text { A14.2.01.036 } & \text { Rr. palpebrales }\end{array}$

Long ciliary nerves

Posterior ethmoidal nerve

Anterior meningeal branch

Anterior ethmoidal nerve

Internal nasal branches

Lateral nasal branches

Medial nasal branches

External nasal nerve

Infratrochlear nerve

Palpebral branches
Nn. ciliar es largos

N. etmoidal posterior

R. meníngeo anterior

N. etmoidal anterior

$\mathrm{Rr}$. nasales internos

Rr. nasales laterales

Rr. nasales mediales

R. nasal externo

N. infratroclear

Rr. palpebrales

\section{DISCUSIÓN}

De la revisión realizada en la literatura anatómica y tomando como base la descripción propuesta por algunos autores clásicos del siglo XIX, el nervio etmoidal anterior se describe como el ramo terminal medial del nervio nasal (nasociliar), el cual, luego de haber ingresado en la cavidad orbitaria entre los orígenes del músculo recto externo del ojo, superior al nervio óptico, se dirige por la pared medial de la órbita ósea hasta el foramen etmoidal anterior para cruzarlo y llegar a la cavidad craneal, atravesando la lámina cribosa del hueso etmoides y llegando a la cavidad nasal, en donde emite dos ramos: uno medial, que se distribuye por la mucosa del tabique nasal, y otro lateral, que se dirige por las paredeslaterales de la mucosa y llega a inervar también los tegumentos del ala y punta nasal. Por su parte, el nervio infratroclear se origina en el foramen etmoidal anterior, se dirige hacia adelante por debajo de la tróclea del músculo oblicuo superior del ojo, llega al ángulo medial del ojo en donde se comunica con el nervio supratroclear y proporciona ramos para el saco y carúncula lagrimal, conjuntiva, tegumentos de los párpados superior e inferior y nariz (Knox, 1831; Wilson, 1855; Gray, 1858; Jones, 1894).

En la primera Nomina Anatomica se puede observar los términos en latín dedicados a los ramos del nervio nasociliar (Fig. 1).

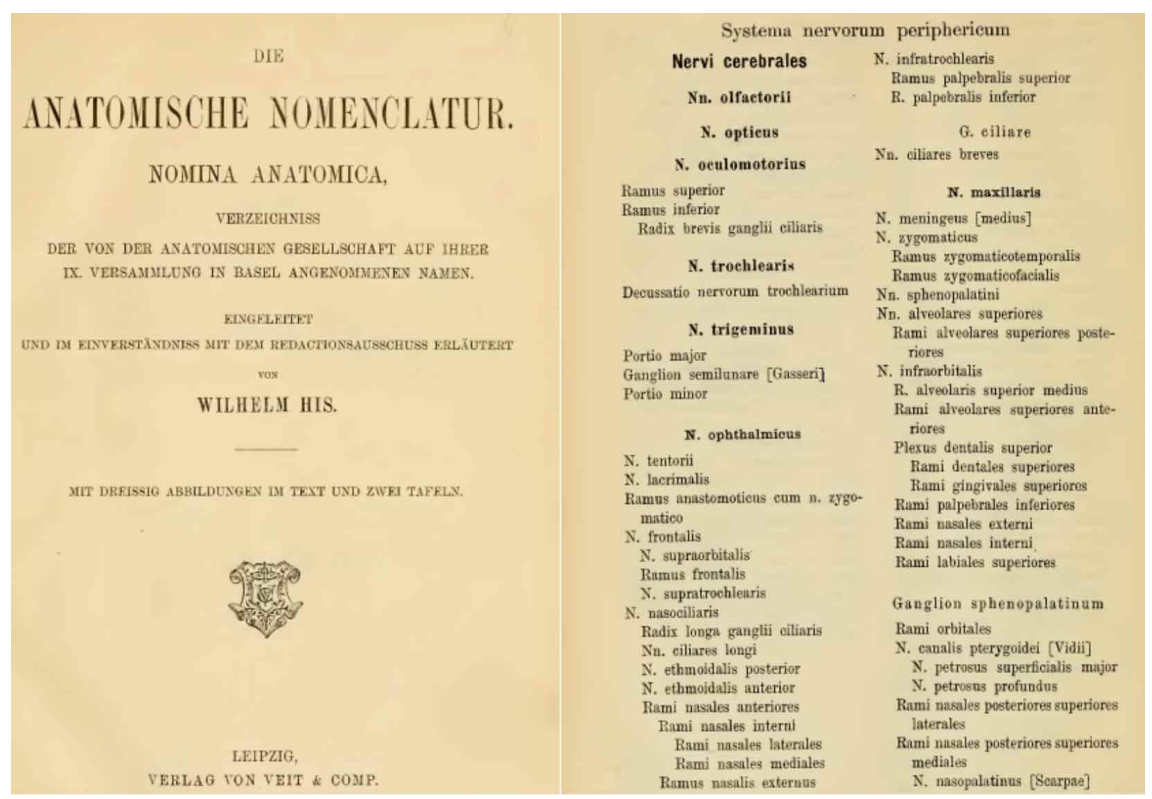

Fig. 1. Portada y página 88 de Nomina Anatomica Basilea, publicado en 1895 (Open Library, 2018). 
Esta Nomina Anatomica tiene errores gramaticales y de descripción en cuanto a la nominación en latín de los términos asociados a los ramos derivados del nervio nasociliar; si tomamos en consideración la descripción que los autores clásicos realizaron sobre ellos, es decir, un ramo nasal "interno" y un ramo nasal "externo" derivados del nervio etmoidal anterior (conocido como nervio nasal interno); por tanto, correspondería para el sustantivo "ramo" el caso nominativo, el género masculino, el número singular, de la segunda declinación, el término en latín para este sustantivo sería ramus; para el adjetivo "nasal" correspondería el caso nominativo, género masculino, número singular, de la tercera declinación, por lo que el término en latín para este adjetivo sería nasalis ya que los adjetivos de segunda clase terminados en consonante como "consul" se flexionan por la tercera declinación; y, en relación al adjetivo "interno" sigue las mismas reglas gramaticales que el sustantivo ramo, por tanto se denominaría con el término latín internus y externus respectivamente.

Es importante indicar que en los textos clásicos de anatomía del siglo XIX consultados no se describen ramos nasales anteriores derivados directamente del nervio etmoidal anterior, como lo da a entender la organización jerárquica del nervio nasociliar de la primera Nomina Anatomica, sino que estos se derivan del ramo nasal externo del ramo etmoidal anterior, conocido como nasal interno (Jamain et al., 1874).

Nomina Anatomica y Terminologia Anatomica conserva el error de número gramatical en latín en el término correspondiente al ramo nasal interno (Dauber, 2006), pese a que los autores clásicos anatomistas del siglo $\mathrm{XX}$ describieron a este ramo como número singular (Lockhart et al., 1965; Testut \& Latarjet, 1978; Rouvière \& Delmas, 2006; Standring, 2008), sin embargo, otros autores no especificaron si se trata de uno o varios ramos nasales internos (Leong, 1986; Latarjet \& Ruiz Liard, 2010; Velayos, 2012; Moore et al., 2013; Pró, 2014). Shankland (2001) describe tres ramos originados del nervio etmoidal anterior: un ramo septal, un ramo lateral y un ramo anterior o superficial.

Es posible también que se haya usado inapropiadamente el genitivo en el caso de "rami nasales interni" y se haya preservado desde la primera Nomina Anatomica, y como una solución compensatoria, toda la expresión se transformó en el plural con el uso del adjetivo, que es lo que suele ocurrir según Marecková et al.

\section{CONCLUSIONES}

Por la evidencia señalada, se propone cambiar el término que tiene el código A14.2.01.031 a ramus nasalis internus observando la pertenencia al caso nominativo, género masculino, número singular de la segunda declinación para los términos ramus e internus; y al caso nominativo, género masculino, número singular de la tercera declinación para el término nasalis.

Además, se propone cambiar el término que tiene el código A14.2.01.036 a ramus palpebralis superior observando la pertenencia al caso nominativo, género masculino, número singular de la segunda declinación para ramus; caso nominativo, género masculino, número singular de la tercera declinación para el término palpebralis y superior; y en estas mismas características gramaticales del latín, incluir el término ramus palpebralis inferior.

Debe haber sido una tarea muy ardua para His y sus colaboradores recopilar toda la información existente en esa época sobre las estructuras anatómicas descubiertas y descritas en diversas partes del mundo y en diferentes idiomas, y además organizarlas de tal manera que se tenga una estructura normalizada y común para todos los anatomistas. Si revisamos en la actualidad los textos de anatomía clásicos, descriptivos, topográficos, funcionales y clínicos, podemos apreciar que aún persisten descripciones diferentes de una estructura, tal como ha sido presentado en este estudio.

GUERRERO, M.; OCAMPO, J.; MOYA, M. P. \& GUERRERO, C. Semantic and grammatical analysis of Latin terms of the terminal branches of the nasociliary nerve. Int. J. Morphol., 37(1):71-75, 2018.

SUMMARY: The need to unify criteria regarding the names of anatomical structures has been a permanent concern of anatomists worldwide. Therefore, and beginning in 1895 a standardization and normalization process of world anatomical terminology was initiated. The Nomina Anatomica is published in an attempt to name the structures with a single name in Latin and the eponyms and homonyms are deleted. Today the Terminologia Anatomica replaces the Nomina Anatomica, with the same characteristics, but with the addition of the term in the language of each country. Nevertheless, some errors persist from the Nomina Anatomica that remain in Terminologia Anatomica, derived from both the Latin grammatical structure, mainly in the number and gender, as well as the description of some anatomical structures. This is the case of the nasociliary nerve branches, specifically the anterior ethmoidal branch and the infratroclear branch. For this purpose, a review of the description of the nasociliary nerve and its terminal branches was made, they were compared between each other, and with the names that appear in the Terminologia Anatomica, to verify that both the description and the Latin grammatical construction are correct. Errors were found in the grammatical and hierarchical structure of the internal nasal branch, as well as the suppression of the upper and lower palpebral branches of the infratrochlear nerve. Therefore, we propose the change of the coded term with A14.2.01.031 to "Ramus nasalis internus" and the addition of the names "Ramus palpebralis superior" and "Ramus palpebralis inferior."

KEY WORDS: Terminologia Anatomica; Nasociliary nerve; Anterior ethmoidal nerve; infratrochlear nerve. 


\section{REFERENCIAS BIBLIOGRÁFICAS}

Acuña, M.; Sinagra, A.; Pérez, M.; Macchia, E.; Manganiello, S. \& Conesa, H. Acerca de la Terminología Anatómica. Rev. Neurocir., 9(4):114-7, 2007.

Álvarez Guisbert, O. \& Campohermoso Rodríguez, O. Evolución histórica conceptual de la Terminología Anatómica. Rev. Cuad., 52(1):113-7, 2007.

Comité Federativo sobre Terminología Anatómica; International Federation of Associations of Anatomists \& Sociedad Anatómica Española. Terminología Anatómica. Terminología Anatómica Internacional. Madrid, Médica Panamericana, 2001.

Dauber, W. Feneis Nomenclatura Anatómica Ilustrada. $5^{\text {a }}$ ed. Barcelona, Masson, 2006.

Duque Parra, J. E.; Gómez Arias, N. C. \& Giraldo Ríos, D. P. Nomenclatura Anatómica Internacional. ¿Un horno microondas en el interior de un volcán activo?. Med. U. P. B., 21(1):43-55, 2002.

Echeverría Pereda, E. \& Jiménez Gutiérrez, I. La terminología anatómica en español, inglés y francés. Panace@, 11(31):47-57, 2010.

Fabry, P.; Baud, R.; Burgun, A. \& Lovis, C. Amplification of Terminologia Anatomica by French language terms using Latin terms matching algorithm: a prototype for other language. Int. J. Med. Inform., 75(7):542-52, 2006.

Gray, H. Anatomy, Descriptive and Surgical. London, John W. Parker \& Son, 1858.

His, W. Anatomische Nomenclatur. Leipzig, Verlang Von Veit \& Comp., 1895.

Jamain, A.; Beaunis, H. É.; Bouchard, A. \& de Batista, R. C. Tratado elemental, de anatomía descriptiva y de preparaciones anatómicas. $2^{\mathrm{a}} \mathrm{ed}$. Madrid, C. Bailly-Bailliere, 1874.

Jones, Q. Human Anatomy. Vol. II. Philadelphia, Lea and Blanchard, 1894.

Knox, R. A System of Human Anatomy. $2^{\text {a }}$ ed. Edinburgh, Maclachlan and Stewart, 1831.

Latarjet, M. \& Ruiz Liard, A. Anatomía Humana. 4ª ed. Vol. I. Buenos Aires, Médica Panamericana, 2010.

Leong, S. K. An Introduction to the Human Nervous System. Singapore, University Press, National University of Singapore, 1986.

Lockhart, R. D.; Hamilton, G. F. \& Fyfe, F. W. Anatomía Humana. Ciudad de México, Interamericana, 1965.

Marecková, E.; Simon, F. \& Cerveny, L. On the new anatomical nomenclature. Ann. Anat., 183(3):201-7, 2001.

Moore, K. L.; Dalley, A. \& Agur, A. Anatomía con Orientación Clínica. $7^{\text {a }}$ ed. Barcelona, Wolters Kluwer Health, 2013.

Open Library. Sitio Web. Internet Archive, 2018. Disponible en: https:// openlibrary.org/

Pró, E. A. Anatomía Clínica. 2a ed. Buenos Aires, Médica Panamericana, 2014.

Rouvière, H. \& Delmas, A. Anatomía Humana, Descriptiva, Topográfica y Funcional. 11a ed. Barcelona, Masson, 2006.

Shankland, W. E. The trigeminal nerve. Part II: the ophthalmic division. Cranio, 19(1):8-12, 2001.

Standring, S. Gray's Anatomy. The Anatomical Basis of Clinical Practice. $40^{a}$ ed. New York, Elsevier - Health Sciences Division, 2008.

Testut, L. \& Latarjet, A. Tratado de Anatomía Humana. Vol. I. Barcelona, Salvat Editores S. A., 1978.

Vandaele, S. \& Harvey, M. G. Les nomenclatures anatomiques. Histoire et traduction.Panace@, 14(38):222-34, 2013.

Vásquez, B. \& del Sol, M. Terminologia Anatomica and Terminologia Histologica. A meeting point between morphologists. Int. J. Morphol., 33(4):1585-90, 2015.
Velayos, J. L. Anatomía de la Cabeza. Barcelona, Médica Panamericana, 2012.

Whitmore, I. Terminologia Anatomica: new terminology for the new anatomist. Anat. Rec., 257(2):50-3, 1999.

Wilson, E. A System of Human Anatomy. Philadelphia, Blanchard and Lea, 1885.

\section{Dirección para correspondencia: \\ Dr. Marco Guerrero Figueroa \\ Facultad de Ciencias Médicas \\ Universidad Central del Ecuador \\ Avenida Sodiro N14-121 e Iquique \\ Quito \\ ECUADOR}

Email: aguerrero@uce.edu.ec

Recibido : 14-08-2018

Aceptado: 03-12-2018 